\title{
Barreras para la institucionalización de la extensión universitaria: Experiencia de la Universidad Nacional, Costa Rica
}

\author{
Barriers to the Institutionalization of University Extension: Experience of the Universidad \\ Nacional, Costa Rica
}

\section{Barreiras à institucionalização da extensão universitária: experiência da Universidade Nacional, Costa Rica}

Resumen: Los retos actuales de las universidades públicas exigen que se fortalezca su misión social estatutaria, donde la extensión universitaria destaca como clave. La incoherencia de la gestión académica, en muchas universidades, restringe dicha integración por vacíos conceptuales, metodológicos y operativos presentes en el proceso de extensión. El presente artículo muestra los resultados de una investigación cualitativa, mediante un estudio de caso, realizada en la Escuela de Planificación y Promoción Social de la Universidad Nacional de Costa Rica, con el objetivo de explorar las barreras universitarias que perturban la contribución de la educación superior al cuarto objetivo de desarrollo sostenible de Naciones Unidas: lograr una educación de calidad, inclusiva, equitativa y con ampliación de oportunidades para más personas. 
http://doi.org/10.15359/ree.25-1.21

http://www.una.ac.cr/educare

educare@una.ac.cr

Palabras claves: Extensión universitaria latinoamericana; gestión académica; universidad pública; educación superior.

\begin{abstract}
The current challenges demand that public universities' statutory social mission be strengthened, where the university extension stands out as key. The incoherence of academic management in many universities restricts this integration due to conceptual, methodological, and operational gaps existing in the extension process. This article shows the results of a qualitative research project, through the case study method, conducted in Escuela de Planificación y Promoción Social (School of Planning and Social Promotion) of the Universidad Nacional de Costa Rica; it aimed to explore the university barriers that disturb the contribution of higher education to the fourth United Nations Sustainable Development Goal: to achieve quality, inclusive, and equitable education with expanded opportunities for more people.
\end{abstract}

Keywords: Latin American university extension; academic management; public university; higher education.

Resumo: Os desafios atuais das universidades públicas exigem o fortalecimento da sua missão social estatutária, onde a extensão universitária é fundamental. A incoerência da gestão académica em muitas universidades restringe a integração por vazios conceptuais, metodológicos e operativos presentes no processo de Extensão. O presente artigo mostra os resultados de uma pesquisa qualitativa, mediante o estudo de caso, realizada na Escuela de Planificación y Promoción Social da Universidade Nacional de Costa Rica, com o objetivo de explorar as barreiras universitárias que dificultam a contribuição da educação superior ao quarto Objetivo do Desenvolvimento Sustentável das Nações Unidas: lograr uma educação de qualidade, inclusiva, equitativa e com ampliação de oportunidades para mais pessoas.

Palavras chaves: Extensão universitária latino-americana; gestão académica; universidade pública; educação superior.

\title{
Introducción
}

La universidad de hoy, considerada célula cultural dela sociedad, en un contexto globalizado y desigual que irrespeta el medio social y ambiental, está llamada a responder a problemáticas de naturaleza diversa. De ahí que la cultura por preservar, desarrollar y promover, desde las misiones de sus respectivos procesos sustantivos universitarios (docencia-investigaciónextensión), deban realizarse desde una amplia concepción. Esto implica, lograr vínculos sociales estrechos universidad-sociedad desde la interdisciplinariedad, la integración horizontal de los conocimientos científicos y sociales y los valores en las formaciones de sus profesionales, por lo que se convierten en los compromisos para orientar a la sociedad hacia el equilibrio de las dimensiones social, económico (no economicista) y ambiental para el sostenimiento de la vida y la protección del planeta. Por tanto, una serie de desafíos socioambientales justifican dar prioridad a la integralidad en la universidad y su gestión académica en la actualidad. 
http://doi.org/10.15359/ree.25-1.21

Santos (2005) advierte a las universidades públicas sobre las problemáticas prioritarias socioambientales desatendidas y los desafíos universitarios de difícil superación mientras persistan sus contradicciones vigentes.

A pesar de la misión social asumida con la Reforma de Córdoba (1918), las universidades latinoamericanas, al igual que otras universidades del mundo, dan énfasis a criterios de corte empresarial y definen sus objetivos ajustados a los intereses del sistema económico dominante (Boni Aristizábal y Gasper, 2011; Walker, 2012). No obstante, dentro de los objetivos de desarrollo sostenible (ODS) de Naciones Unidas, se demanda a las universidades que logren una educación de calidad, inclusiva, equitativa y con ampliación de oportunidades para más personas. Así, hoy se detectan cambios vitales en la tercera función, tanto conceptuales como metodológicos y operativos, con el fin de poder transitar hacia una universidad contextocéntrica (Núñez Paula, 2002) y asumir el modelo de extensión alternativo o crítico (Tommasino, 1994; Tommasino et al., 2006), que contemple, dentro de la formación, conocimientos, destrezas y valores que demanda la humanidad (Walker y Mc Lean, 2013). Condiciones a las que se puede aspirar con la integración de los procesos sustantivos universitarios en contexto (Menéndez, 2016; Monge Hernández et al., 2018; Tünnermann Bernheim, 1998). Esto, a criterio de los autores, exige que las universidades institucionalicen el carácter de función y proceso de la extensión (González, 1996, 2016), identifiquen su gestión específica en el trabajo sociocultural universitario (González Fernández Larrea, 2002), definan la integralidad como su esencia (González Moreno, 2006) y mantengan la comunicación universidad-sociedad (Freire, 1993) como vía explícita para orientar las tres funciones académicas e impactar, de manera crítica y positiva, la sociedad.

Las universidades mantienen la extensión vigente, como es el caso de la Universidad Nacional de Costa Rica (UNA) que sostiene una importante cantidad de programas, proyectos y actividades de extensión en las comunidades nacionales, en contextos urbanos, rurales, fronterizos, costeros. No obstante, Zlateva Peneva (2017) revela que en el ecosistema institucional de la UNA "Los desafíos de la extensión se relacionan con la carencia de prioridad institucional y presupuestaria, la falta de valoración y reconocimiento académico" (p. 65).

En las universidades del Sur global, sea en Sudáfrica, Centro o Suramérica, los discursos del cuerpo docente extensionista coinciden en que impera un ambiente adverso de distinto orden y naturaleza, que va desde dificultades para acceder a transporte institucional y financiar la logística de las actividades (hospedaje, alimentos), hasta recibir igualdad de reconocimientos y valoraciones académicas intelectuales de los productos de la extensión, respecto a los obtenidos desde la investigación (Monge Hernández et al., 2019; Sutz, 2010; Zlateva Peneva, 2017).

En el caso de Costa Rica, si bien la extensión universitaria y la acción social de las universidades públicas, ambas se constituyen en un componente estratégico de la educación superior en el Consejo Nacional de Rectores (CONARE), coexisten en un contexto adverso de valoración y voluntad política universitaria poco efectiva respecto a los trabajos académicos con 
http://doi.org/10.15359/ree.25-1.21

http://www.una.ac.cr/educare

educare@una.ac.cr

las comunidades, en estas y para estas, con los sectores populares y el tejido social de base (Consejo Nacional de Rectores [CONARE]. Oficina de Planificación de la Educación Superior [OPES], 2016). Esto dificulta la permanencia en las comunidades y pone en riesgo el cumplimiento de los compromisos asumidos en los ODS y en la misión de las universidades públicas costarricenses.

Se entiende que la extensión es fuente de aprendizajes, enseñanzas y generación de conocimientos críticos transformacionales, procedentes de las interacciones y diálogos entre los diversos actores sociales (Tünnermann Bernheim, 1998) y es la función que mejor expresa la condición ética política de las universidades y su labor educacional, científica y cultural (Tommasino y Cano, 2016); por su lado, la extensión crítica permite"una amplia gama de prácticas desarrolladas en el medio rural latinoamericano, que tienen su origen en una visión crítica de la estructura social" (Tommasino, 1994, p. 13). Un proceso en el que la comunidad universitaria y las personas de las comunidades se convierten en aprendientes entre sí, cuando se integran saberes técnicos y populares para entender críticamente las realidades y afrontar en conjunto los retos que impone el contexto (Freire, 1997). Por tanto, los sujetos participantes encuentran medios para superar las condicionantes históricas dominantes, hegemónicas y de poder que privan la libertad de ser de las personas (Freire, 2005). Una práctica pedagógica resulta básica para generar un proceso educativo que humanice al estudiantado, para que, en su futuro ejercicio de la profesión, se desarrolle a partir de los cambios transformativos que potencien sus aportaciones al bien común y sus compromisos y acciones para la construcción de un mundo mejor.

A la luz de los presupuestos teórico-metodológicos anteriores y las contradicciones del CONARE y la UNA, se presenta esta investigación de las barreras de gestión universitaria que perturban el cumplimiento de dichos objetivos globales e institucionales. Se asume una investigación cualitativa tomando como método fundamental el estudio de caso de la Escuela de Planificación y Promoción Social (EPPS) de la UNA. Esta investigación aporta a la comprensión de las posibilidades reales del cuerpo docente para la integración de las tres funciones académicas sustantivas y mantener su presencia en las comunidades costarricenses. Estas barreras institucionales constituyen desafíos universitarios para asegurar la calidad de la educación superior y su contribución a la sociedad.

Para mostrar los resultados de la presente investigación, inicialmente se exponen los fundamentos de la extensión universitaria latinoamericana y las características del caso de estudio, para ofrecer los criterios metodológicos utilizados. Seguido, se presentan los principales hallazgos relacionados con las problemáticas que el cuerpo docente de EPPS expresó enfáticamente, tomando como fuente principal las entrevistas aplicadas. Asimismo, a la luz de las reflexiones con este grupo de docentes y de los textos revisados (Escuela de Planificación y Promoción Social [EPPS], 2016), se develaron barreras para incrementar la concordancia de la gestión académica y administrativa con los requerimientos de la extensión universitaria. Finalmente, se enuncian las conclusiones más relevantes en relación con aspectos de la gestión académica de la UNA que afectan el desarrollo de la extensión y la permanencia del personal docente extensionista y estudiantes en las comunidades de Costa Rica, así como las necesidades de mayor flexibilidad y simplificación administrativa para sostener la comunicación con la sociedad. 
http://doi.org/10.15359/ree.25-1.21

\section{El porqué de la extensión universitaria en la universidad latinoamericana}

La Reforma de Córdoba en 1918 generó una revolución en la dinámica general de las universidades públicas y la academia, hito distintivo de la universidad de Latinoamérica y el Caribe. Este proceso permitió, entre muchos otros beneficios, aspectos básicos para el desarrollo de la sociedad, mediante el desenvolvimiento de la universidad y el surgimiento de la conciencia crítica (Tünnermann Bernheim, 1998). Esta reforma ratificó los principios base de organización de la universidad, construida desde la convicción de lograr una propuesta educativa con identidad y defensa de América Latina (Tünnermann Bernheim, 1998). A partir de un proyecto de ley universitaria, se sentaron las nuevas bases estatutarias para las universidades públicas argentinas que, posteriormente, se difundieron por el resto de Latinoamérica y el Caribe.

Según Tünnermann Bernheim (1998), a esta Reforma le antecede un contexto de descontento e insatisfacción social con las universidades existentes, por su carácter colonial, clasista, excluyente de ciertas razas y clases sociales y reproductoras de los sistemas de dominación. Es categorizada como el primer intento de cambio de dirección de la universidad Latinoamericana y el punto de ruptura de la universidad tradicional que la presionaba a mantener el poder político y económico y apoyar los intereses de las clases dominantes. A lo interno, se logró una concepción tripartita de la integración de la comunidad universitaria, gobernada por medio de la elección. Se establecieron derechos vitales de autonomía universitaria, democratización de la educación; asimismo, mecanismos de inclusión y participación, desde la gratuidad de la educación, la contratación de cargos profesionales por concurso de oposición, la asistencia y docencia libre, la periodicidad de cátedra, divulgación de actos universitarios y el sistema diferenciado de organización de las universidades, así como la extensión universitaria y la asistencia social estudiantil.

La Reforma permitió dar un giro de la universidad hacia las comunidades, al designar "la misión social de la universidad y su participación en el estudio de los problemas nacionales" (Tünnermann Bernheim, 1998, p.118).

En la década de 1960, la extensión experimentó uno de sus mejores ascensos con el modelo pedagógico concientizador, a partir de las ideas de la izquierda latinoamericana y las contribuciones brasileñas, con el pensamiento base de la educación popular y la educación transformadora, de Pablo Freire, Darcy Ribeiro y otros; la extensión consiguió un importante giro ontológico y epistemológico al incorporar objetivos de creación de conciencia y construcción de conocimientos de manera horizontal, dialógica y liberadora (Serna Alcántara, 2007). Desde entonces, la extensión ha buscado participar en acciones sociales transformadoras, con posicionamientos políticos anti hegemónicos que contribuyan al despertar de las conciencias, de las capacidades de análisis crítico y de acciones colectivas de la sociedad. Estos procesos adicionan, además, mejoras sustantivas a la formación estudiantil y favorecen la recuperación y promoción de la identidad latinoamericana y sus luchas sociales.

A pesar de los avances alcanzados, a cien años de la Reforma de Córdoba, permanecen 
http://doi.org/10.15359/ree.25-1.21

http://www.una.ac.cr/educare

educare@una.ac.cr

incoherencias, un sin número de desafíos, para que la universidad logre completar su proceso formativo inclusivo, justo e integral desde las tres funciones académicas. Si bien se han propuesto cambios institucionales y se han logrado avances, persisten debilidades históricas; como Tünnermann Bernheim (1978) señala, "Pero en la práctica poco es lo que se logra: la universidad sigue siendo señorial, frecuentada exclusivamente por los hijos de las clases dominantes y controlada por abogados y clérigos" (p. 95).

Por eso, la extensión continúa siendo clave para la universidad y la sociedad, para contribuir a formar mejores profesionales, la producción de conocimientos valiosos para la sociedad, la realimentación de la docencia y la investigación y, entre otros resultados, dar un aporte sustantivo a quienes más lo necesitan (Universidad de la República. Extensión, 2010). De ahí que resulta indiscutible el potencial de la extensión en función de la democratización de los conocimientos y en la promoción de la democracia, la participación y la justicia social, abriendo oportunidades de acceso a las poblaciones más vulnerables y conectando sus necesidades con la investigación y la formación de profesionales.

El carácter holístico y multidimensional de la extensión se refleja en sus dimensiones. Menéndez (2016) propone cinco dimensiones de la extensión, agrupadas en: I) académica e institucional, II) social, cultural y productiva, III) comunicacional y dialógico, IV) pedagógica y V)

Figura 1: Dimensiones de la extensión universitaria desde la experiencia de la Universidad Nacional de Litoral (UNL), Argentina

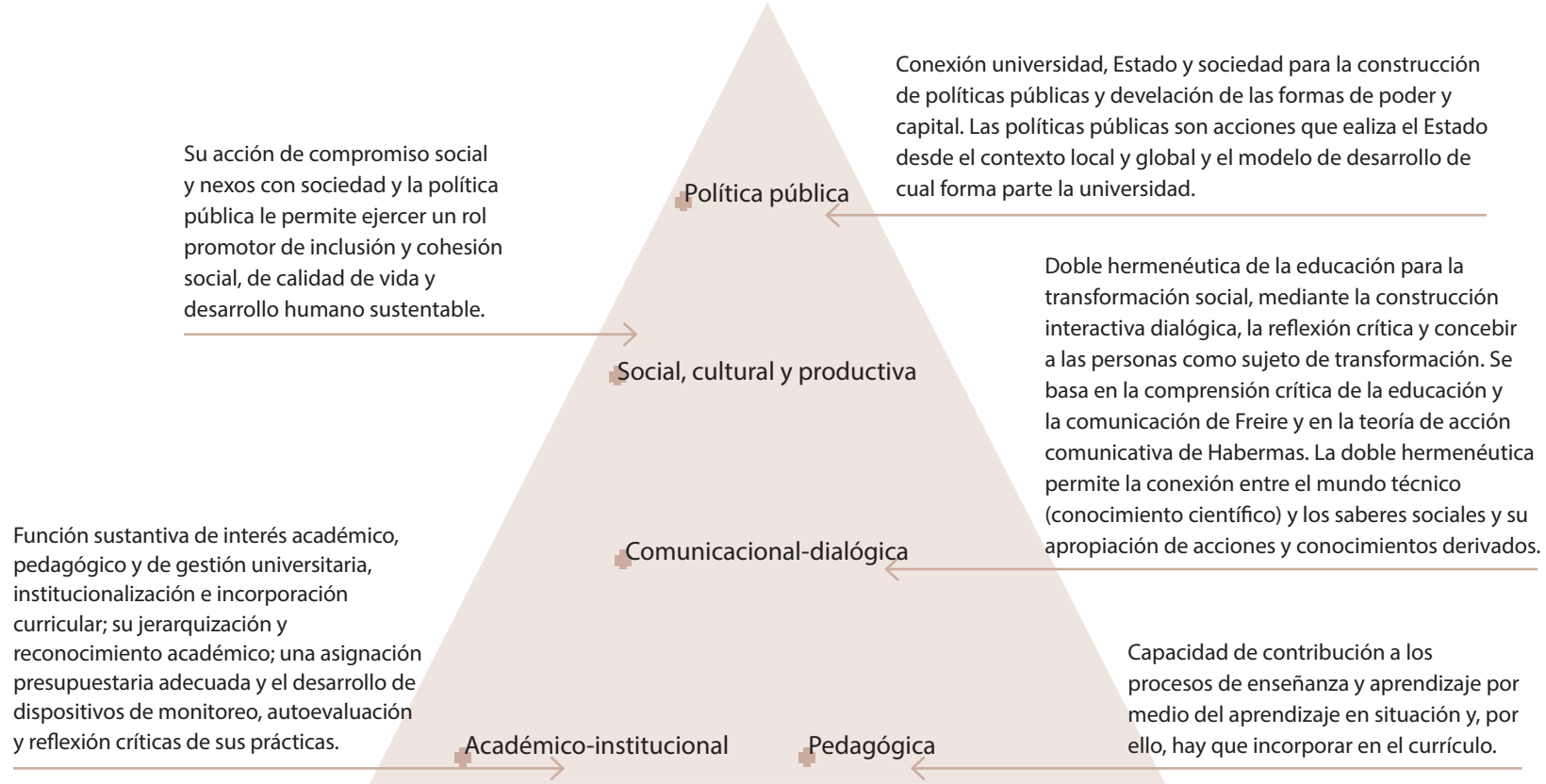

Nota: Elaboración propia a partir de Menéndez (2016). 
http://doi.org/10.15359/ree.25-1.21

política (Figura 1). Si las universidades públicas pretenden resultados positivos en todas esas dimensiones, en la institución debe necesariamente preponderar un clima de gestión interno que asegure, en su praxis, esas dimensiones en las comunidades, por estas y para estas, y los logros en los diversos espacios, necesidades y sujetos involucrados, según lo esperado.

\section{La extensión universitaria en la UNA, Costa Rica}

En Costa Rica, la soberanía y la autonomía universitaria está garantizada en la Constitución Política de la República de Costa Rica de 1949, artículo 84 (Asamblea Nacional Constituyente, 1949). De igual manera, el Plan Nacional de la Educación Superior Universitaria Estatal (PLANES) 2016-2020 de CONARE, que integra las cinco universidades públicas, se propone: "fortalecer la interacción de la universidad con la sociedad, en un proceso permanente, participativo y planificado que responda a los requerimientos de la realidad" (CONARE. OPES, 2016, p. 81). Para lograr ese objetivo, cada universidad orienta el desarrollo de proyectos de extensión y acción social y desarrolla proyectos interinstitucionales financiados con la convocatoria anual de Fondos del Sistema.

Por su parte, la UNA posee un ideario social distintivo adscrito a su lema de Universidad Necesaria con amplia experiencia de procesos con las comunidades, mediante programas, proyectos y actividades (PPAA) de extensión universitaria en todo el país. Asimismo, algunas unidades académicas, como es el caso de la EPPS (Monge Hernández et al., 2018), presentan en su plan de estudio la gestión curricularizada de la extensión. De ahí que la UNA conciba la extensión universitaria en los siguientes términos:

Procesos de creación y desarrollo de las capacidades de sus actores sociales, institucionales y locales, y de transformación social integral para una mayor calidad de vida de las comunidades. La extensión nutre la formación integral del académico y del estudiante y fortalece el compromiso que demanda el desarrollo humano. Comprende acciones conjuntas sociedad-universidad, continuas y planificadas, para la generación de una mejora social integral y la retroalimentación del quehacer universitario. (UNA, Consejo Académico, 2009, p. 6)

El Estatuto Orgánico vigente de la UNA contiene principios que enfatizan en valores vinculados a la extensión: "humanismo..., inclusión..., conocimiento transformador...", entre otros (Asamblea Universitaria de la Universidad Nacional [UNA], Costa Rica, 2016, p. 12). A pesar de la pertinencia de esos procesos en las comunidades, por estas y para estas en contextos en desarrollo, las realidades son que el cuerpo docente extensionista (en adelante cuerpo extensionista) afronta importantes desafíos para mantener su presencia en las regiones (Monge Hernández et al., 2019; Zlateva Peneva, 2017). 
http://doi.org/10.15359/ree.25-1.21

http://www.una.ac.cr/educare

educare@una.ac.cr

La burocracia, madre de la pobre dinámica operativa, y las desigualdades en la asignación de recursos financieros, afecta la visión estratégica humanista e integradora de la universidad; entorpece posibles alternativas de solución a las problemáticas ambientales y de aportación al desarrollo humano de los grupos más vulnerables del país. Contrariamente, con el título de la autonomía que la Constitución Política de Costa Rica otorga a sus universidades públicas, la gestión universitaria genera cada vez más reglamentos y normativa administrativa que devienen en barreras y restricciones para el desarrollo de la extensión.

Una situación similar se genera con PLANES-CONARE, con desigualdades en la planificación de los proyectos extensionistas versus investigación. Si bien no se presentan cifras económicas de las inversiones, emergen asimetrías cuando las metas a proyectos universitarios del área de investigación de PLANES se duplican respecto a las de proyectos de extensión. Ejemplo de ello son los proyectos financiados con la convocatoria de 2016 de Fondos del Sistema, que estimaba la aprobación de 32 proyectos de extensión, mientras que se sumaban 67 para investigación (CONARE. OPES, 2016).

La extensión universitaria es un proceso continuo y permanente con muy valiosos resultados en la formación integral y el desarrollo humano del estudiantado, entre lo que se destacan las capacidades de sensibilidad y conciencia sobre las realidades de las personas con desigualdad social, el incremento de la resiliencia y reflexividad emocional, el fortalecimiento de valores de beneficios para la sociedad y en habilidades profesionales diversas (Monge Hernández et al., 2018). A las comunidades que participan en dicho proceso les aporta en la comprensión y aplicación de capacidades esenciales para su desarrollo humano, relacionadas con las habilidades y conocimientos prácticos, liderazgo, cohesión social, empoderamiento en proyectos comunitarios, y acceso a servicios y derechos humanos, entre otros (Monge Hernández et al., 2019).

Todo lo anteriormente fundamentado, mediante análisis documental y el estudio de bibliografía a científica, exige una investigación más a fondo que identifique las barreras más relevantes que enfrenta el cuerpo extensionista de EPPS en su gestión actual de extensión, como desafíos a la educación superior, en el cumplimiento de los ODS hacia una educación de calidad, inclusiva, equitativa y con ampliación de oportunidades para más personas.

\section{Metodología utilizada en la investigación}

Esta investigación se realizó desde la epistemología interpretativa, con un carácter exploratorio, que permite dar interpretación a las realidades observadas. Corbetta (2007) señala la prioridad de conocer la individualidad de la persona y su forma de ver el mundo, para comprender la realidad social y las categorías mentales (interpretación, percepción, sentimientos, esperanzas, motivaciones, actos y concepciones del mundo) de las personas, con respecto a lo estudiado. 
http://doi.org/10.15359/ree.25-1.21

En esta investigación, la entrevista y la observación participante buscaron disponer de datos empíricos, mediante la consulta a los sujetos participantes (cuerpo extensionista) y observación participante (prácticas extensionistas desarrolladas en comunidades costarricenses), con el fin de conocer sus perspectivas vinculadas a la temática investigada.

Se aprovechó la experiencia acumulada de EPPS que, desde su creación en 1974, incorporó la extensión en el plan de estudios (Monge Hernández et al., 2018), que se explican más adelante, y es pionera en el desarrollo de PPAA de extensión. Según los expedientes consultados, acumularon 28 PPAA de extensión durante 2002-2017; 14 de ellos integrados (docencia, investigación y extensión) y los restantes de vinculación externa, principalmente apoyados, financieramente, por otras instituciones públicas de desarrollo social (EPPS, 2016).

El programa estudios de Planificación y Promoción Social tienen cuatro asignaturas enfocadas a lograr las interacciones del estudiantado con los sectores más vulnerables del país: Práctica Organizativa I, Práctica Organizativa II, Práctica de Formulación y Evaluación de Proyectos I y Práctica de Formulación y Evaluación de Proyectos II; con seis créditos cada uno, en el tercer y cuarto año de la carrera (EPPS, 2016). Dentro de sus objetivos figuran la búsqueda de diálogos, construcciones y aprendizajes conjuntos estudiantes y sujetos locales en las comunidades, que se constituyan en espacios de aprendizaje, enseñanza y transformaciones sociales mediante abordajes epistemológicos de concepción constructivista. Solo con estas

Tabla 1: Compendio de métodos de recolección de datos y su propósito

\begin{tabular}{|c|c|}
\hline Técnica & Propósito \\
\hline $\begin{array}{l}\text { Estudio de bibliografía } \\
\text { científica }\end{array}$ & $\begin{array}{l}\text { Fundamentar teórica y metodológicamente el proceso, función y gestión de la } \\
\text { extensión universitaria como postulados de partida }\end{array}$ \\
\hline Revisión documental & $\begin{array}{l}\text { Vincular los compromisos estatutarios y políticas con la extensión universitaria de } \\
\text { la UNA. Fundamentar procesos y limitaciones de la extensión universitaria de EPPS. }\end{array}$ \\
\hline Entrevistas a profundidad & $\begin{array}{l}\text { Analizar a nivel criterial no intencional las principales barreras que impiden que el } \\
\text { cuerpo extensionista de EPPS desarrolle acciones de extensión. }\end{array}$ \\
\hline Grupos de discusión & $\begin{array}{l}\text { Analizar conscientemente las principales barreras que impiden que el cuerpo } \\
\text { extensionista de EPPS priorice la extensión. }\end{array}$ \\
\hline Observación participante & $\begin{array}{l}\text { Observar experiencias, relaciones y significancias de la extensión para el cuerpo } \\
\text { extensionista de EPPS. }\end{array}$ \\
\hline $\begin{array}{l}\text { Triangulación de } \\
\text { informantes y documentos }\end{array}$ & Cotejar la información recopilada para fortalecer la constatación del resultado. \\
\hline
\end{tabular}

Nota: Elaboración propia. 
http://doi.org/10.15359/ree.25-1.21

http://www.una.ac.cr/educare

educare@una.ac.cr

asignaturas del plan de estudios se puede vincular a 90 estudiantes, anualmente, con distintas comunidades y organizaciones nacionales (EPPS, 2016).

Se realiza esta investigación a partir de técnicas seleccionadas pertinentemente, considerando la experiencia, en el segundo semestre de 2017 (Tabla 1).

El análisis documental permitió revisar el Estatuto Orgánico de la UNA y su política, respecto a la extensión universitaria de EPPS en la UNA. Tanto las entrevistas como el grupo de discusión se centraron en conocer las problemáticas administrativas-académicas (logística administrativa, recursos económicos, vehículos, equipos, materiales pedagógicos) que más les desmotiva y restringe las interacciones con las comunidades, incluyendo lo que afecta al sector estudiantil participante y la información se validó con la observación participante realizada en las comunidades involucradas, durante la ejecución de las asignaturas.

En total, se realizaron: un grupo de discusión y seis entrevistas a profundidad con personas del cuerpo extensionista (cuatro mujeres y dos hombres), con jornada parcial o total en extensión. Ambas técnicas se aplicaron en el campus Omar Dengo de la UNA, los meses de agosto y setiembre del 2017, específicamente al cuerpo extensionista que imparte las asignaturas de Práctica Organizativa I y II, quienes en su mayoría responden a la extensión universitaria curricularizada (Monge Hernández et al., 2018).

El grupo consultado fue seleccionado a partir del criterio experto, para lo cual se eligieron personas que poseen más de 5 años de experiencia en extensión (dentro o fuera de la universidad). Para asegurar el anonimato de los sujetos informantes, no se emplean códigos de género. La revisión documental integró información no publicada de informes y ponencias, normativas institucional y nacional y documentación vinculada con los PPAA. Se utiliza el registro de los criterios ofrecidos sobre la gestión académica y del personal docente en asignaturas que integran la extensión universitaria y las acciones propias de programas, proyectos y actividades de extensión, realizados en los últimos quince años.

La observación participante se hizo para constatar en la práctica la información obtenida de informantes y otros documentos. Se desarrolló entre junio y setiembre de 2018, en las comunidades rurales costarricenses, duranteacciones correspondientes a la Práctica Organizativa II. La conjugación de información, mediante la triangulación, fortaleció la comprensión de las narrativas de las personas entrevistadas, en la construcción de las-barreras más relevantes que expresa el cuerpo extensionista como principales resultados de la investigación. Se siguieron criterios de credibilidad a partir de la recolección de información suficiente para documentar la investigación, así como la transcripción de las entrevistas y las anotaciones de las observaciones. La triangulación de la información permitió la verificación de visiones y hechos. Se ofreció anonimato y consentimiento informado a sus participantes. 
http://doi.org/10.15359/ree.25-1.21

\section{Resultados y discusión}

Se presentan las principales limitaciones de la gestión académica que perturban el proceso de extensión universitaria, asumidas como barreras que impiden disponer de una universidad pública justa e inclusiva, orientada a la consecución de la integralidad en la formación profesional y de la transformación social (comunicación universidad-sociedad), demandada por sociedad actual y el planeta.

Las categorías utilizadas para mostrar los hallazgos y su discusión son: gestión académica, extensión universitaria latinoamericana, y restricciones institucionales para el aprovechamiento de integralidad de las funciones académicas y la multidimensionalidad de la extensión.

\section{Amenazas desde la gestión académica, administrativa y financiera}

\section{-Burocracia administrativa institucional}

El cuerpo extensionista, participante de esta investigación, entendió el derecho a disponer de canales de apoyo y de facilidad en los trámites para el uso de los recursos financieros necesarios para desarrollar la extensión. Expresaron contrariedades y desgaste provocado por los excesivos requisitos administrativos institucionales para realizar el ejercicio de la extensión. La mayoría consideró que existe falta de comprensión y apoyo administrativo sólido requerido para que las gestiones institucionales respondan a las necesidades de los procesos universitarios comprometidos y demandados por las comunidades y los sectores con que se trabaja. Si bien sus contratos no son para gestiones administrativas, frecuentemente relegan las actividades académicas, pues la burocracia administrativa les obliga. En palabras de Extensionista_3, esto hace que tengamos que asumir actividades administrativas con el fin de poner peso frente al funcionario administrativo y lograr que nos den una respuesta más rápida y oportuna en los trámites que necesitamos.

Esa situación provocó que el cuerpo extensionista no solo se hiciera cargo de la función académica (docente, extensionista e investigativa) y de comunicación con las comunidades que le competen, sino también se responsabilizara de una gran cantidad de trámites administrativos. Trámites que, además, le impusieron excesiva regulación de plazos y especificidades difíciles de cumplir por los proyectos extensionistas. El Extensionista_3 explicó que uno de los de factores de más desgaste y desmotivación ha sido atender la gestión de los recursos financieros, porque la misma universidad tiene estipulado procesos que no entronizan con la planificación de giras (salidas de campo) y las realidades presupuestarias del proyecto. Este extensionista también indicó:

En el Departamento de Financiero indican que, para brindar los apoyos para las giras del estudiantado, debe realizarse un proceso en el que se les deposite el dinero en su cuenta. El monto se calcula según viáticos estudiantiles y no se permite que los excedentes sean devueltos al proyecto. Esto genera inconsistencias en los presupuestos, ya que en muchos casos esos 
http://doi.org/10.15359/ree.25-1.21

http://www.una.ac.cr/educare

educare@una.ac.cr

excedentes ayudan a financiarse las próximas giras o refrigerios para las actividades en las comunidades. Si bien hay estudiantes que nos apoyan todo el año, hay otros que hacen actividades muy específicas en un corto plazo, por ejemplo, la ayuda que nos ofrecen estudiantes de práctica de sociología, y las gestiones de sus ayudas económicas hay que realizarlas con mínimo un mes de antelación, cuando no tenemos aún claridad de su trabajo.

A su labor académica, se le adicionaron constantemente actividades de liquidación de gastos de servicios y materiales para lograr las interacciones con las comunidades, especialmente de zonas alejadas de la gran área metropolitana (GAM). Hay servicios que no pudieron ser pagados, porque las organizaciones locales no tenían comprobantes de pago en las calidades formales que exige el Departamento de Financiero. Por ejemplo, del pago de una panga (pequeñas embarcaciones artesanales utilizadas en zonas costeras o ríos de Costa Rica) para llegar a una isla o a un territorio indígena, usado para el desplazamiento del cuerpo universitario y las personas locales participantes (de bajos recursos) hasta los puntos de encuentro; asimismo, el pago de refrigerios elaborado por miembros de las organizaciones, hospedajes en comunidades rurales alejadas, materiales comprados en esas comunidades o insumos locales necesarios para generar los diálogos, dar capacitaciones o seguimiento de actividades planificadas.

Si bien los recursos que ingresan a la universidad provienen del pueblo, a la hora de devolver algo a las comunidades (sea mediante el pago de la panga, ayudas para el transporte de grupos sociales, aporte de refrigerio o materiales didácticos, semillas o insumos productivos afines al abordaje, otros) se presentan, a lo interno de la institución, cuestionamientos de si a la UNA le corresponde financiar esos costos y si existe optimización de la asignación de recursos en el desarrollo de PPAA. En opinión de Extensionista_5, en la UNA han prevalecido desigualdades entre las aprobaciones y disponibilidades, casi incuestionadas, de las inversiones en investigación con compras de materiales y equipos sumamente costosos. En ocasiones, durante las evaluaciones de PPAA, a extensionistas se les ha cuestionado el porqué de las asignaciones presupuestarias hasta en rubros muy básicos para ofrecer condiciones dignas y recursos mínimos necesarios para los procesos sociales y comunitarios. Es frecuente que se les señale dar asistencialismo, cuando intentan dotar de inversiones básicas para las interacciones con las personas que menos tienen, mas nunca se escuchan cuestionamientos sobre los resultados de las inversiones en PPAA de investigación o de su apropiación por parte del sector empresarial. Así, a lo interno de la universidad no se obstaculiza el aporte universitario hacia el sector productivo del país, pero se desmerita el trabajo social y los reconocimientos académicos asignados por las contribuciones a las labores extensionistas y de las ciencias sociales. Esto puso en evidencia la orientación empresarial (Serna Alcántara, 2007), emergente en las universidades públicas, que desestimula los procesos extensionistas, al tiempo que presiona a favor de los intereses hegemónicos y de sectores minoritarios privilegiados. 
http://doi.org/10.15359/ree.25-1.21

Según las observaciones de Extensionista_5, resultó incoherente lo estipulado cuando promocionamos el desarrollo local, pues se impide [al cuerpo extensionista] que se adquieran, por ejemplo, los refrigerios elaborados por las mismas mujeres o agrupaciones sociales con las que trabajamos, que además son muchos más sanos que los productos industrializados que se compran en la región central. Señalaron que, en ocasiones, prefieren comprar los refrigerios en las comunidades y pagarlos de su propio bolsillo (Extensionista_5 y Extensionista_6).

\section{-Inoperante motilidad hacia las comunidades y en estas}

En el tema logístico y administrativo, otro inconveniente se relacionó con las condiciones, movilizaciones y desplazamientos requeridos para los encuentros entre participantes de las actividades extensionistas a favor de las transformaciones sociales. El cuerpo extensionista solicitó mejorar el sistema de programación y asignación de vehículos institucionales para llegar a las comunidades y hacer posible la interacción universidad-comunidad, de encuentros de saberes. Además, consideró prioritario que la UNA consiga flexibilizar los trámites de manera que compatibilicen con las demandas docentes, pactadas con la comunidad. En este sentido, requieren mejoras en las posibilidades de generar una agenda sólida de investigación y extensión permanente desde la atención de las necesidades de las comunidades nacionales y para estas (Extensionista_6). Por otro lado, si bien tanto la gestión de la UNA, desde las unidades académicas, como en EPPS, históricamente, han hecho esfuerzos para adquirir vehículos institucionales, el Departamento de Transportes gestiona con agendas y normas que no responden a los tiempos, las demandas y las realidades de las comunidades ni de los procesos académicos conexos.

El extensionista responsable debe tramitar las giras con plazos sumamente amplios, lejanos a la dinámica comunal y de las organizaciones vinculadas (...) las giras se programan con un mes de antelación (...) Si se utiliza el vehículo descentralizado no hay seguros que garanticen su seguridad y la de las personas en caso de accidente (...) Cuando se ha enfrentado un accidente, la cantidad de trámites, la lentitud y los procesos son sumamente engorrosos y desgastantes (Extensionista_1)

Los casos de los vehículos institucionales descentralizados, de las unidades académicas, son otra limitación no resuelta, pues la UNA no dispone de suficientes choferes institucionales contratados $y$, aunque el cuerpo extensionista acepte conducirlos, las pólizas institucionales de riesgo de accidentes de tránsito no le protegen.

A criterio de Extensionista_2, a la UNA, se le han presentado otras condiciones en contra que son parte de la naturaleza del proceso de extensión. Por ejemplo, surgieron complicaciones de gestión universitaria en casos de cancelación o modificación de las salidas al campo, por razones 
http://doi.org/10.15359/ree.25-1.21

http://www.una.ac.cr/educare

educare@una.ac.cr

ajenas a su control, como el tener que trasladar a las poblaciones locales a otras localidades, por decisión comunitaria, condiciones climáticas, problemas en la disponibilidad de tiempo, modificaciones de fechas, tipo de vehículo o número de participantes que viajan a atender las actividades. La extensión ameritó, además, integrar constantemente a personal académico o estudiantes de otras disciplinas o carreras para el tratamiento integral e interdisciplinar de las problemáticas locales. Al Extensionista_3 le preocupó esa restricción, porque considera fundamental la agilidad institucional para garantizar la presencia del cuerpo extensionista en las comunidades, pues en ocasiones, ante la ausencia del equipo de la UNA, las comunidades cancelan las reuniones y se interrumpe la planificación y la secuencia de las actividades, sus participantes (estudiantes, funcionariado de otras instituciones, miembros de las organizaciones locales) bajan su motivación, se pierde la confianza y se obstaculizan los procesos sociales y comunitarios planificados, según lo pudimos constatar durante la observación participante. Ante lo expuesto, la mayoría de extensionistas coincidió en la importancia de contar con algún status de prioridad en la asignación de transporte institucional en valoración y reconocimientos a sus trabajos. Argumentan que, cuando deben asistir a las comunidades de manera impostergable, se ven obligados a invertir más tiempo y esfuerzos, al tener que utilizar los transportes públicos o el vehículo familiar, con sus propios recursos (Extensionista_5).

Las dificultades en transportes ocupan lugar cimero en la discusión sobre factores que restringen el desarrollo de la extensión, pues les limita las planificaciones académicas y los tiempos y compromisos efectivos de trabajo con las comunidades. Los grupos de extensionistas que han tenido únicamente jornada de investigación o extensión, sin carga laboral de docencia, tienen aún más limitaciones, porque carecen de ese requisito esencial de aprobación de giras con categoría de prioridad de asignación de vehículo institucional. Esto les generó más desgaste por cuanto tuvieron que innovar para encontrar a una persona docente dispuesta a figurar como responsable y tramitar las solicitudes de giras (salidas a terreno), en el sistema, para gestionar el transporte institucional y, una vez aprobadas, realizar el cambio de responsable. Luego de superado este proceso, cuando llega el día del traslado a campo, el cuerpo extensionista alegó que, es muy probable, le corresponda hacer frente al mal humor de los choferes, sus bromas, falta de cortesía y desidia durante las giras, [también reconocen que] aunque no es el común, hay choferes que se consideran un miembro más de las comunidades y participan en los talleres y opinan (Extensionista_2). Otra de las personas consultadas mencionó la impertinente actitud que presentan algunos choferes, aspecto poco motivador cuando han tenido que vencer todos los trámites anteriores.

Si bien las personas extensionistas de la UNA aspiran a aprovechar la multidimensional de la extensión universitaria (Menéndez, 2016), en beneficio de la sociedad costarricense y la coproducción de saberes, la gestión institucional universitaria les absorbe gran parte de su productividad, energía, motivación y tiempo. 
http://doi.org/10.15359/ree.25-1.21

\section{- Dificultades económicas para la presencia estudiantil}

Otra preocupación recurrente mostrada por los grupos extensionistas durante el grupo de discusión y en las entrevistas aplicadas, fue la incertidumbre sobre los recursos económicos y materiales para garantizar la permanencia universitaria, la participación estudiantil y la sostenibilidad de los procesos sociales y comunitarios locales. La urgencia de recursos para cubrir los costos de traslados y estancia de los estudiantes en las comunidades son una preocupación que [nos] amenaza todo el año (Extensionista_2). Si bien una parte de docente y estudiantes contaban con el apoyo financiero del Fondo para el Fortalecimiento de las Capacidades Estudiantiles (FOCAES), del Programa Aula Móvil de CONARE y de contrapartidas voluntarias, en hospedaje y alimentación principalmente, de organizaciones locales e instituciones como el Instituto de Desarrollo Rural (INDER), esos recursos y colaboraciones resultaron insuficientes para dar apoyo a la totalidad del estudiantado que permanece anualmente en las comunidades. Algunos grupos de estudiantes que no logran disponer de redes ni apoyo institucional y trabajan el proceso extensionista desde su plan de estudios (EPPS, 2016), con organizaciones muy pequeñas, no tienen la oportunidad de recibir ayudas económicas o becas estudiantiles para solventar sus traslados. De igual manera, estudiantes con problemas socioeconómicos tienen dificultades para financiar los costos de alimentación, traslados y materiales básicos que utilizan durante los procesos participativos en las comunidades, para estas y con estas. Esto refleja la limitación vigente en la UNA para integrar la dimensión pedagógica (Menéndez, 2016), y el cumplimiento del principio de igualdad en la participación estudiantil en prácticas de extensionistas incorporadas al currículo para la totalidad de las carreras universitarias.

Por otra parte, el cuerpo extensionista percibió un ambiente de competición y de presión institucional por la búsqueda de los recursos o ventas de servicios para poder apoyar los costos de los procesos sociales y comunitarios, y las ayudas estudiantiles necesarias para llevar adelante los proyectos de extensión emergentes desde las comunidades, debido a que la UNA tiene recursos económicos muy limitados para tales fines y se generan vínculos y compromisos éticos ineludibles con las comunidades. El cuerpo extensionista entrevistado y participante del grupo de discusión reconoció que, en la UNA, abundan discursos que estimulan la búsqueda de ingresos para sostener las actividades académicas, con el ofrecimiento de servicios de manera remunerada, como consultorías, venta de servicios y oferta de posgrados pagos. Por tanto, se evidencia que avanza un sistema que presiona hacia la privatización del espacio público y restringe los fines de las universidades públicas. A las universidades, al ser parte del Estado, les corresponde ser garante de que las personas, sin ningún distingo, accedan a sus servicios.

Extensionista_6 recomendó a la UNA generar espacios que promuevan la participación estudiantil mediante la asignación de más presupuesto institucional y otros fondos estudiantiles concursables o fuentes de recursos alternativos para incentivar la realización de procesos sociales y comunitarios, con fines de transformación social, principalmente fuera del GAM. De igual 
http://doi.org/10.15359/ree.25-1.21

http://www.una.ac.cr/educare

educare@una.ac.cr

manera, el grupo de extensionistas expresó su aspiración de contar con estudiantes asistentes que apoyen los proyectos de extensión e investigación, tanto para favorecer su formación como para promover la actividad académica y social. Sin embargo, la participación estudiantil en la extensión se torna cada vez más difícil, tanto por aspectos económicos, como logísticos y trámites burocráticos excesivos. Según Extensionista_6: son tormentosos la fila de trámites y cuestionamientos que hay que superar para que más jóvenes[universitarios] permanezcan [durante su carrera]en las comunidades.

\section{-Insuficiente integración de la dimensión administrativa}

La extensión universitaria se diferencia de los procesos de docencia e investigación:

La extensión universitaria como función totalizadora en la Universidad, implica a toda su estructura y recursos humanos, requiere de una estrecha coordinación de los factores internos y externos que participan en el cumplimiento de los programas y proyectos propuestos y su organización debe desarrollarse desde la perspectiva del beneficiario, a partir de generar un movimiento que logre involucrar y comprometer a toda la comunidad universitaria y a representantes de las entidades laborales vinculadas a la formación de los profesionales y líderes extrauniversitarios. (González Fernández Larrea, 2002, p. 70)

Al decir González Moreno (2006) que la integralidad es la esencia de la extensión, significa que no es solamente un atributo que cualifica la extensión. Esto se evidenció en el criterio que plantea la demanda por parte del cuerpo extensionista de un mayor apoyo de la Vicerrectoría de Extensión y de la gestión de la unidad académica para diseñar los proyectos de extensión e investigación integrales, en los lugares donde desarrollan las prácticas de asignaturas en el plan de estudios de la carrera. Estas orientaciones pueden ser un mecanismo de búsquedayasignación de recursos financieros para garantizar la presencia continua, progresiva y planificada del proceso extensionista presente en las comunidades. Por ejemplo, pueden utilizarse para concursos de fondos internos en la UNA, en CONARE o mediante la coordinación interinstitucional, con otras universidades e instituciones públicas, para que financien la curricularización de la extensión o proyectos integrales completos de largo plazo en las comunidades (y se cumplan las expectativas generadas en las personas locales) y no solo se quede como la práctica anual pedagógica de un número reducido de estudiantes (que se ajusta al periodo lectivo). Es necesario, también, que las vicerrectorías dispongan de recursos financieros y de tiempos académicos para un mayor desarrollo de los proyectos de investigación y extensión. Sería ideal hasta que ellos nos busquen y ofrezcan oportunidades de colaboración (Extensionista_6).

\section{- Conflictos en el proceso de desarrollo profesional extensionista}


http://doi.org/10.15359/ree.25-1.21

El desarrollo profesional presentó limitaciones en tres aristas: I) necesidades de capacitaciones académicas, II) valoración académica de su proceso en las comunidades, con estas y para estas, y III) excesivas cargas de trabajo administrativo que dificultan la productividad académica y las condiciones de vida del extensionista. Si bien la formación integral de estudiantes se logra mediante interacciones más profundas, planificadas y sistemáticas con personas que tienen otras realidades, hay dificultades para realizar este proceso. El comprender y estudiar las problemáticas de las realidades permite ampliar capacidades humanas necesarias para los ejercicios profesionales éticos que necesita el país y la humanidad (Monge Hernández et al., 2018). De ahí, la demanda de más capacitaciones para fortalecer la extensión, sobre los aspectos filosóficos, el alcance, nuevos enfoques, metodologías, empoderamiento social, etc., y que podamos orientar a nuestros estudiantes y aportar a llegar a una verdadera práctica integral en comunidad (Extensionista_6).

El cuerpo extensionista consultado solicitó mayores iniciativas que promuevan la sistematización, con apoyo de equipos externos que pueden ser suministrados por las vicerrectorías, para elevar la integralidad de las funciones académicas y la legitimación de la extensión, mediante reflexiones teóricas y vínculos con la investigación y la docencia. Asimismo, potenciar la sostenibilidad y calidad de la extensión que desarrollan en las comunidades. Sin embargo, pidió programarse en fechas y horarios accesibles a la mayor parte de extensionistas. En palabras de Extensionista_5: hay que generar cambios en la programación universitaria pues los cursos de desarrollo profesional, regularmente son programados los jueves y viernes, días que por agenda académica están dedicados a las giras de campo.

Por otro lado, el cuerpo extensionista alertó que los procesos sociales y comunitarios demandan de esfuerzos y tiempos que exceden las jornadas académicas. El desarrollo de acciones con la modalidad de PPAA y, adicionalmente, las cuatro asignaturas de las prácticas, con 90 estudiantes por año, amerita participación, coordinación y monitoreo con las comunidades durante la construcción de diagnósticos, estrategias y labores de seguimiento a las acciones locales. La docencia combinada con la extensión/investigación demanda arduos procesos académicos y transformaciones sociales que, además, son mal calificados en términos de aportes intelectuales, de acuerdo con las evaluaciones del régimen de carrera académica (Consejo Universitario de la Universidad Nacional [UNA] Costa Rica, 2015).

Por tanto, el cuerpo extensionista considera que los procesos sociales y comunitarios no son vistos como labores académicas, a pesar de lo establecido en Estatuto Orgánico vigente de la UNA, el legado institucional extensionista, los resultados sumamente valiosos para la formación (Monge Hernández et al., 2018), la investigación y el abordaje de problemáticas sociales. Por ejemplo, en la UNA, aunque una ponencia en un congreso internacional tiene un rango de valoración entre 0 y 4 puntos (Consejo Universitario de la UNA, Costa Rica, 2015), la puntuación asignada a las personas consultadas no excede los 0,5 puntos, la valoración más repetitiva fue 0,33 por ponencia, sin importar el tipo de congreso, país o recuperación de 
http://doi.org/10.15359/ree.25-1.21

http://www.una.ac.cr/educare

educare@una.ac.cr

conocimiento logrado. De igual manera, en el grupo de discusión, florecieron desacuerdos por la valoración que reciben los artículos publicados del área de extensión. Resaltaron las desigualdades con que se valoran las producciones de las ciencias sociales, respecto a otras disciplinas vinculadas con las ciencias exactas; asunto que han logrado conocer, mediante cotejo informal de las calificaciones que reciben sus colegas de otras unidades académicas. Consideran contraproducente para la institución y la sociedad costarricense que lo producido mediante el paradigma constructivo interpretativo o de investigación acción participativa de las ciencias sociales reciba puntuaciones muy por debajo de las producciones realizadas desde el paradigma positivista o post positivista. Llama la atención que, aunque los artículos sean evaluados por pares externos como requisito de publicación por parte de las revistas, a lo interno de la UNA, sus evaluadores universitarios otorgan una nueva valoración. Los grupos extensionistas advirtieron del desconocimiento y subvaloración, por parte de estos sistemas evaluadores, sobre los paradigmas de las ciencias sociales y de la riqueza de la ecología de saberes, de la investigación acción y de su pertinencia ética, política y pedagógica de esos procesos universitarios para la sociedad.

En Carrera Académica tampoco se le han valorado los premios nacionales otorgados al mérito del trabajo social y comunitario, lo que de nuevo muestra diferencias respecto a los reconocimientos por la investigación. Las personas extensionistas externaron su frustración, mediante expresiones como, Los extensionistas vivimos con el poco reconocimiento académico que tienen las actividades que realizamos (Extensionista_3); Para el Extensionista_4, los trabajos de extensión no se aprecian institucionalmente en su real valor. El Extensionista_1 dijo que, mientras el producto de una investigación recibe buen puntaje en carrera académica, no sucede lo mismo con nuestros productos de extensión, aunque un plan estratégico o uno de desarrollo local implique cantidad de horas extras al ser participativo [y aportes significativos al desarrollo y la política nacional]. Los argumentos dados, plantearon muchos retos de gestión académica por delante y pusieron en evidencia la importancia de lograr una atmósfera de credibilidad y valoración de los trabajos de extensionistas y del estudiantado y de los PPAA que se realizan en las comunidades y entender que todos los sujetos (universitarios y de las comunidades) son poseedores de conocimientos valiosos.

\section{- Insuficiente reconocimiento de la extensión universitaria}

Ante la adversidad en la cultura institucional de la UNA, se debe reforzar la identidad del personal administrativo y académico con la extensión. El personal universitario ha de interiorizar, de manera oportuna, la responsabilidad ética con la misión social universitaria, el fondo filosófico y político de la función extensionista. El cuerpo extensionista aseguró que, a lo interno de la universidad se carece de una comprensión integral, ética y humanista sobre las realidades que enfrentan las comunidades alejadas de GAM y de los objetivos y responsabilidades de las 
http://doi.org/10.15359/ree.25-1.21

prácticas extensionistas. La universidad, al desconocer y debilitar esta función, permite que las lógicas administrativas impongan cada día más barreras para que, tanto el personal académico como sus estudiantes, profundicen en las acciones de comunicación dialógicas, reflexivas y colectivas, prioritarias para nuestra sociedad frente al contexto neoliberal globalizado vigente.

El extensionismo de la UNA aspira a una comunidad universitaria que, en su totalidad, se integre y se sientan parte de la contribución social que realiza la UNA y del aporte al desarrollo de la sociedad (Extensionista_5). Para ello, han de promoverse cambios institucionales sustanciales a lo interno de la UNA; según Extensionista_4: para que la extensión prospere lo mínimo necesario es que logremos que tenga un rango similar al de investigación.

En el ámbito administrativo, el personal universitario desconoce los compromisos y responsabilidades [de la extensión] que exceden lajornada de trabajo para la quesenos ha contratado (Extensionista_4). Extensionista_1 propuso que los cuerpos de funcionarios colaboradores de Transportes, Seguridad, Financiero, Asesoría Jurídica y Proveeduría Institucional, así como jefaturas de dichos departamentos, sean convocados a participar de procesos de los PPAA de extensión, en comunidades rurales, por parte de la Vicerrectoría de Extensión, para que consigan comprender las realidades académicas, geográficas, climáticas, socioculturales y territoriales con las que se enfrentan las comunidades, que comparten sus saberes, valores y cultura con el cuerpo extensionista y estudiantil. De esta manera, promover que el personal administrativo se informe y se sensibilice con los compromisos éticos-políticos institucionales y las responsabilidades que le corresponde a toda la comunidad universitaria.

La intención fundamental perseguida por extensionistas es generar una institucionalidad que apunte a un sistema de gestión más flexible, más sensibilizado y comprometido con las finalidades de la extensión, para la atención de las prioridades que demandan la sociedad y el planeta, por un desarrollo humano sustentable. El grupo extensionista expresó su aspiración a que estas acciones sean valoradas por las universidades como prioritarias y estratégicas, dada su contribución a la sociedad desde la construcción de capacidades, formación integral de futuro estudiantado egresado y oportunidades de bienestar a favor de las poblaciones que menos poseen.

El cuerpo extensionista hizo un llamado a toda la comunidad universitaria para unirse y aportar a los logros sociales, principalmente en beneficio de las personas más empobrecidas, marginadas, excluidas u oprimidas, en comunidades costarricenses olvidadas de las instituciones y las políticas públicas.

La extensión universitaria de la UNA, desde su fundación en 1974, ha permitido a las comunidades rurales y urbanas costarricenses mantener a la universidad actualizada, con sus saberes, así como democratizar el conocimiento. La extensión permite dar acceso al conocimiento, mediante la participación de niñas y niños, jóvenes, personas adultas mayores; mujeres organizadas; grupos obreros; grupos de pequeñas unidades de producción agrícolas, 
http://doi.org/10.15359/ree.25-1.21

http://www.una.ac.cr/educare

educare@una.ac.cr

ganaderas y de pesca locales; representantes de organizaciones locales de base y movimientos sociales, entre muchos otros. Así, considerando la naturaleza de extensión universitaria, esta función presenta el mayor potencial para que las universidades públicas latinoamericanas aporten al cumplimiento de los ODS en su objetivo de garantizar una educación superior de calidad, inclusiva, equitativa y con mayores oportunidades de acceso a las personas.

\section{Orientaciones de mejoras según resultados de la exploración}

En el Estatuto Orgánico de la UNA (Asamblea Universitaria de la UNA, Costa Rica, 2016), figuran los principios UNA de transparencia en la rendición de cuentas y probidad. El primero busca garantizar el cumplimiento de la misión institucional, el uso eficiente y una gestión responsable de los recursos asignados. Este principio ofrece tribuna para paliar los límites que en la gestión académica de la UNA se develan en la presente exploración como perturbadores de la gestión extensionista; mientras que la probidad señala que todos los sujetos universitarios poseen el deber de actuar con honestidad y rectitud en el ejercicio de los derechos y deberes otorgados por la UNA, así como la debida administración y tutela de los recursos públicos bajo su responsabilidad. Si el artículo 84 de la Constitución Política costarricense otorga a las universidades la libertad para definir su organización y para la UNA, significa que existen potencialidades para realizar cambios académicos e institucionales, que reviertan los obstáculos institucionales vigentes, antes expuestos, para lo que es condición obligada la integración del conocimiento científico con el saber popular (Menéndez, 2016), y la integración de los procesos académicos sustantivos y los métodos dialógicos y participativos que se empleen para promover la transformación social, y garantizar la transparencia y rendición de cuentas del destino de los recursos públicos.

\section{Conclusión}

En la gestión académica de la UNA existen barreras importantes que se establecen ante el proceso de extensión universitaria, al obstaculizar el trabajo con las comunidades, en estas y para estas. Las barreras resultantes, por su denominación, exponen su naturaleza administrativa y académica, a saber: la burocracia en los procesos administrativos, la subvaloración académica e institucional de la extensión, la desmotivación institucional para el desarrollo de proyectos extensionistas. Esto se manifiesta, sobre todo, en las grandes dificultades para desarrollar y cubrir las inversiones básicas de las prácticas extensionistas que se impulsan desde el plan de estudios de la carrera de Planificación y Promoción Social. Tanto los procesos de aprendizaje en las aulas como en la realidad específica latinoamericana de nuestras comunidades son mecanismos necesarios para la formación de profesionales con criticidad, sensibilidad, creatividad y propuestas ante los problemas de desarrollo que viven las personas que poseen menos oportunidades $y$, por tanto, espacios que aportan sustantivamente al cuarto objetivo de los ODS.

Esta investigación logró mostrar las barreras inherentes a un mundo fuertemente 
http://doi.org/10.15359/ree.25-1.21

presionado por el modelo económico dominante, sus perspectivas de capital humano y las visiones neoliberales e instrumentales respaldadas por diversas entidades internacionales. Estas barreras, que perturban la gestión de la extensión, afectan intrínseca e ineludiblemente la integralidad de los procesos sustantivos universitarios; por ello, se torna relevante el enrutamiento de estrategias institucionales que aporten a procesos de construcción participativa y democrática con nuestros pueblos. Por consiguiente, se requiere apuntar a estrategias institucionales efectivas para que, desde un posicionamiento ético, político y pedagógico crítico y transformador, integren la docencia con la investigación y la extensión, sin prevalencia de una sobre otra; de manera que aseguren la flexibilidad y la simplificación para las interacciones universidad-sociedad y consoliden la comprensión de la misión estatutaria, poniendo como centro el sostenimiento de la vida de las personas y la protección del planeta.

\section{Reconocimiento}

Este trabajo se dedica al cuerpo docente extensionista de la UNA, Costa Rica. Nuestro agradecimiento especial la EPPS-UNA, por el apoyo ofrecido a esta investigación y la colaboración en el trabajo previo complementario realizado en el tema de políticas institucionales de extensión. Ese primer esfuerzo, que motivó este artículo, fue presentado en el III Congreso de Extensión Universitaria, UNA: Dialogando en territorios-Concepto y políticas de Extensión 20182028, realizado en noviembre, 2017, en Costa Rica, razón por la cual hacemos extensiva nuestra gratitud a la Vicerrectoría de Extensión-UNA.

\section{Declaración de Material complementario}

Este artículo tiene disponible, como material complementario:

-La versión preprint del artículo en https://doi.org/10.5281/zenodo.3661249

\section{Referencias}

Asamblea Nacional Constituyente. (08 de noviembre, 1949). Constitución Política de la República de Costa Rica.

Asamblea Universitaria de la Universidad Nacional, Costa Rica. (2016). Estatuto Orgánico de la Universidad Nacional. Gaceta Extraordinaria n. 3-2016. http://www.documentos.una. ac.cr/handle/unadocs/3964

Boni Aristizábal, A. y Gasper, D. (2011). La universidad como debiera ser. Propuestas desde el desarrollo humano para repensar la calidad de la universidad. Sistema. Revista de Ciencias Sociales, 220, 99-115. 
http://doi.org/10.15359/ree.25-1.21

http://www.una.ac.cr/educare

educare@una.ac.cr

Consejo Nacional de Rectores. Oficina de Planificación de la Educación Superior. (2016). Plan Nacional de la Educación Superior Universitaria Estatal 2016-2020. Autor. https://www. conare.ac.cr/images/articulos/planes 2016 2020.pdf

Consejo Universitario de la Universidad Nacional, Costa Rica (27 de julio de 2015). Reglamento del régimen de carrera académica. Alcance N.03 a la Gaceta N.o 14-2015. https://www.google.

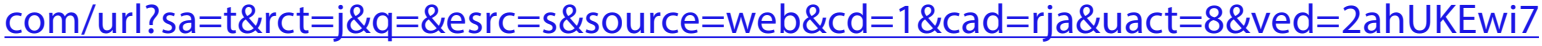
4LS4ulziAhVQOhoKHYW4ATYQFjAAegQIABAC\&url=http\%3A\%2F\%2Fwww.documentos. una.ac.cr\%2Fbitstream\%2Fhandle\%2Funadocs\%2F2877.6\%2FREGLAMENTO\%2520DEL \%2520REGIMEN\%2520DE\%2520CARRERA\%2520ACAD\%25C3\%2589MICA.\%2520NUEV O\%2520EO.doc\%3Fsequence\%3D17\&usg=AOvVaw111nPrp38AA7kKbIK-MZMF

Corbetta, P. (2007). Metodología y técnicas de investigación social. McGraw-Hill.

Escuela de Planificación y Promoción Social. (2016). Compendio de información: Plan estudios vigente, informes anuales 2010-2016, plan estratégico 2011-2016 y programas de asignaturas (Práctica de Formulación y Evaluación de Proyectos I y II y Práctica organizativa I y II). Universidad Nacional.

Freire, P. (1993). ¿Extensión o comunicación? La concientización en el medio rural. Siglo XXI.

Freire, P. (1997). Pedagogía de la autonomía. Siglo XXI.

Freire, P. (2005). Pedagogía del oprimido. Siglo XXI.

González, G. R. (1996). Un modelo de extensión universitaria para la extensión universitaria. Su aplicación a la cultura física y el deporte [Tesis doctoral]. Instituto Superior de Cultura Física "Manuel Fajardo", La Habana, Cuba.

González, G. R. (Febrero, 2016). La extensión universitaria: Promotora del cambio, la innovación y la transformación sociocultural. Conferencia Inaugural. En J. R. Saborido (Presidencia), XIII Taller Internacional de Extensión Universitaria. Taller llevado acabado en el 10mo Congreso Internacional de Educación Superior "Universidad 2016". Ministro de Educación Superior de Cuba. La Habana, Cuba.

González Fernández Larrea, M. (2002). El modelo de gestión de la extensión universitaria para la Universidad de Pinar del Río (Tesis doctoral). Universidad de Pinar del Río “Hermanos Saiz Montes de Oca", Pinar del Río, Cuba.

González Moreno, M. (2006). Formulación teórico-metodológica de la promoción cultural de la investigación para la integración de los procesos universitarios de extensión e investigación en el Instituto Superior Politécnico José Antonio Echeverría [Tesis doctoral]. Centro de Referencia para la Educación de Avanzada, Habana, Cuba 
http://doi.org/10.15359/ree.25-1.21

Menéndez,G.(2016). Desafíos presentey futuro de la extensión camino alos 100 años de la Reforma Universitaria de 1918. Cuadernos de Extensión Universitaria de la UNLPam. 2(2), 13-42. http:// www.unlpam.edu.ar/images/extension/Cuadernos\%20de\%20Extensi\%C3\%B3n\%20 Universitaria\%20de\%20la\%20UNLPam\%20-\%20N\%C2\%BA\%202.pdf

Monge Hernández, C., Mena García, P. y Gamboa Conejo, R. (2018). Resultados de la interacción con la comunidad en la formación universitaria desde el enfoque de las capacidades. Un estudio de caso en la Universidad Nacional de Costa Rica. +E: Revista de Extensión Universitaria, 8(8), 110-125. https://doi.org/10.14409/extension.v8i8.Ene-Jun.7722

Monge Hernández, C., Zlateva Peneva, P. y Boni Aristizábal, A. (2019). Extensión universitaria y aprendizaje-servicio. Análisis de dos casos en Sudáfrica y Costa Rica desde el enfoque de las capacidades para el desarrollo humano. Revista Interamericana de Educación de Adultos, 41(1), 111-137. https://www.crefal.org/rieda/index.php?option=com_content\&v $\underline{\text { iew }=\text { article\&id }=294 \& \text { temid }=242}$

Núñez Paula, I. A. (2002, octubre). Aproximación metodológica para introducir la gestión del aprendizaje en las organizaciones y comunidades. Amiga Versión 2.0. En Gerencia en Ciencia e Innovación (pp. 177-207), GECYT.

Santos, B. de Souza. (2005). La universidad en el siglo XXI para una reforma democrática y emancipadora de la universidad. Universidad Nacional Autónoma de México. https:// estudogeral.sib.uc.pt/bitstream/10316/44179/1/La\%20Universidad\%20en\%20 el\%20Siglo\%20XX1\%20-\%20Para\%20una\%20Reforma\%20Democratica\%20y\%20 Emancipadora\%20de\%20la\%20Universidad.pdf

Serna Alcántara, G. A. (2007). Misión social y modelos de extensión universitaria: Del entusiasmo al desdén. Revista Iberoamericana de Educación, 43(3), 3-25. https://rieoei.org/historico/ deloslectores/1662Aquiles.pdf

Sutz, J. (2010). La integralidad de las funciones universitarias como espacio de preguntas recíprocas. Cuadernos de Extensión, 1, 43-60. http://pim.udelar.edu.uy/wp-content/ uploads/sites/14/2016/11/Cuaderno integralidad.pdf\#page $=44$

Tommasino, H. (1994). Grupos y metodología grupal en la lechería uruguaya: La asistencia técnica en grupos de producción remitentes a Conaprole del departamento de San José (10 informe de investigación, No. 338.1 TOMg). Unidad de Extensión, Facultad de Veterinaria, UDELAR.

Tommasino, H., González, M. N., Grabino, V., Luengo, L. y Santos, C. (2006). Extensión, interdisciplinariedad y desarrollo en el medio rural: El caso de la Colonia Fernández Crespo. En H. Tommasino y P. de Hegedüs. Extensión: Reflexiones para la intervención en el medio urbano y rural (pp. 257-295). Departamento de Publicaciones de la Facultad de Agronomía, Universidad de la República Oriental del Uruguay. http://beu.extension.unicen.edu.ar/ 
http://doi.org/10.15359/ree.25-1.21

http://www.una.ac.cr/educare

educare@una.ac.cr

\section{xmlui/handle/123456789/234}

Tommasino, H. y Cano, A. (2016). Modelos de extensión universitaria en las universidades latinoamericanas en el siglo XXI:Tendencias y controversias. Universidades, 67, 7-24. https:// www.researchgate.net/publication/293952059 Modelos de extension universitaria en las universidades latinoamericanas en el siglo XXI tendencias y controversias

Tünnermann Bernheim, C. (1978). El nuevo concepto de extensión universitaria y difusión cultural y su relación con las políticas de desarrollo cultural en América Latina. Anuario de Estudios Centroamericanos, 4, 93-126. https://revistas.ucr.ac.cr/index.php/anuario/article/ view/3305

Tünnermann Bernheim, C. (1998). La reforma universitaria de Córdoba. Educación superior y sociedad, 9(1), 103-127. http://www.iesalc.unesco.org/ess/index.php/ess3/issue/view/22

Universidad de la República. Extensión. (2010). Extensión en obra. Experiencias, reflexiones, metodologías y abordajes en extensión universitaria. Autor. http://beu.extension. unicen.edu.ar/xmlui/bitstream/handle/123456789/201/Extensi\%c3\%b3n\%20en\%20 obra\%20Experiencias\%2c\%20reflexiones $\% 2 c \% 20$ metodolog\%c3\%adas $\% 20 y \% 20 \% 20$ abordajes\%20en\%20extensi\%c3\%b3n\%20universitaria.pdf?sequence=1\&isAllowed=y

Universidad Nacional, Consejo Académico. (15 de noviembre de 2009). Lineamientos para la gestión de programas, proyectos y actividades. Gaceta N.o 17-2009: Autor. http://www. documentos.una.ac.cr/handle/unadocs/1793

Walker, M. (2012). A capital or capabilities education narrative in a world of staggering inequalities? International Journal of Educational Development, 32(3), 384-393. https://doi. org/10.1016/j.ijedudev.2011.09.003

Walker, M. y McLean, M. (2013). Professional education, capabilities and the public good. The role of universities in promoting human development. Routledge. https://doi. org/10.4324/9780203083895

Zlateva Peneva, P. (2017). La contribución de la extensión universitaria al desarrollo humano y a la expansión de las capacidades. Análisis de proyectos de la Universidad Nacional de Costa Rica. Universidad en Diálogo, 7(2), 33-71. http://dx.doi.org/10.15359/udre.7-2.3 